\title{
CRITICAL MARKOV BRANCHING PROCESSES WITH GENERAL SET OF TYPES
}

\author{
BY \\ H. HERING
}

\begin{abstract}
This paper is concerned with the asymptotic behaviour of critical, quasi-positively regular Markov branching processes. Several results which have been established with restrictions on the set of types or on the parameter are proven on slightly different moment assumptions for an arbitrary set of types and continuous as well as discrete parameter. The methods employed are analytic and rest upon the properties of probability-generating functionals constructed from the given transition function.
\end{abstract}

The asymptotic behaviour of critical, positively regular Markov branching processes with general set of types has already been studied for discrete parameter by Mullikin [9]. For arbitrarily large finite set of types a corresponding continuous parameter theory had previously been given by Čistjakov [1]. A few years later, Joffe and Spitzer [5] weakened the moment assumptions underlying the theory for processes with finite set of types and discrete parameter. In the following a treatment of critical, quasi-positively regular processes (\$1.4) with general set of types is given, which applies to continuous as well as discrete parameter.

\section{Preliminaries.}

1.1. Setup. Every state of the kind of branching system we are interested in may be conceived as a finite population of objects each of which is characterized by a set of properties called its type. Let $X$ be the set of types and $X^{(n)}$ the symmetrization of $X^{n}:=\bigotimes_{i=1}^{n} X_{i}$ with $X_{i}=X$ for $i=1, \ldots, n$. The element $\left\langle x_{1}, \ldots, x_{n}\right\rangle \in X^{(n)}$ may then be regarded as representing the unordered population of $n$ objects with the types $x_{1}, \ldots, x_{n}$ respectively. Denoting by $\theta$ the state in which "no object is present" and setting $X^{(0)}:=\{\theta\}$, we define

$$
\hat{X}:=\bigoplus_{n=0}^{\infty} X^{(n)}
$$

Received by the editors June $1,1970$.

AMS 1969 subject classifications. Primary 6067; Secondary 4690, 4750.

Key words and phrases. Markov brançhing processes, branching transition function, discrete parameter, continuous parameter, generating functionals, generating mappings, analyticity of generating mappings, factorial moment-functionals, expansion of generating functionals, expansion in factorial moment-functionals, moment recurrence relations, moment semigroup, positively regular processes, quasi-positive regularity, critical processes, nonsingular processes, asymptotic extinction, rate of extinction, conditional distribution given nonextinction, limiting conditional distribution. 
as our state space. Let $\mathfrak{A}$ be a $\sigma$-algebra on $X$. Writing $\chi_{A}$ for the indicator function of $A \subset X$ and defining

$$
\begin{aligned}
N(A, \hat{x}): & =0, & \hat{x} & =\theta, \\
& :=\sum_{i=1}^{n} \chi_{A}\left(x_{i}\right), & \hat{x} & =\left\langle x_{1}, \ldots, x_{n}\right\rangle,
\end{aligned}
$$

for $A \subset X,\left\langle x_{1}, \ldots, x_{n}\right\rangle \in \hat{X}$, and $n>0$, the $\sigma$-algebra $\hat{\mathfrak{A}}$ on $\hat{X}$ induced by $\mathfrak{A}$ is identical with the Borel extension of

$$
\left\{\left\{\hat{x} \in \hat{X}: N\left(A_{i}, \hat{x}\right)=n_{i}, i=1, \ldots, m\right\}: A_{i} \in \mathfrak{A}, n_{i} \geqq 0, i=1, \ldots, m, m>0\right\}
$$

where $A_{1}, \ldots, A_{m}$ may be taken mutually disjoint. For details see [8], [2].

Denoting the set of nonnegative integers by $Z_{+}$and the set of nonnegative reals by $\boldsymbol{R}_{+}$, suppose that either $\boldsymbol{T}=\boldsymbol{Z}_{+}$, or $\boldsymbol{T}=\boldsymbol{R}_{+}$, and let $\boldsymbol{P}_{t}(\hat{A} \mid \hat{x})$ be some homogeneous transition function defined for $t \in T_{0}:=T-\{0\}, \hat{A} \in \hat{A}$, and $\hat{x} \in \hat{X}$. That is, we assume that $\boldsymbol{P}_{t}(\hat{A} \mid \hat{x})$ is a probability measure on $\hat{\mathfrak{A}}$ for each $t \in \boldsymbol{T}_{0}$ and $\hat{x} \in \hat{X}$, an $\hat{\mathfrak{A}}$-measurable function of $\hat{x}$ for each $t \in T_{0}$ and $\hat{A} \in \hat{\mathfrak{A}}$, and that it satisfies the Chapman-Kolmogorov equation

$$
\boldsymbol{P}_{t+s}(\hat{A} \mid \hat{x})=\int_{\hat{x}} \boldsymbol{P}_{t}\left(\hat{A} \mid \hat{x}^{\prime}\right) \boldsymbol{P}_{s}\left(d \hat{x}^{\prime} \mid \hat{x}\right)
$$

for $\hat{A} \in \hat{\mathfrak{A}}, \hat{x} \in \hat{X}$, and $t, s \in \boldsymbol{T}_{0}$. If in addition $\boldsymbol{P}_{t}(\cdot \mid \cdot)$ satisfies the condition of branching without immigration

$$
\begin{gathered}
\boldsymbol{P}_{t}\left(X^{(0)} \mid \theta\right)=1, \\
\boldsymbol{P}_{t}\left(\left\{N\left(A_{i}, \hat{x}\right)=n_{i}, i=1, \ldots, m\right\} \mid\left\langle x_{1}, \ldots, x_{k}\right\rangle\right) \\
=\sum_{\substack{n_{i}^{(1)}+\ldots+n_{i}^{(k)}=n_{i} \\
i=1, \ldots, m}} \prod_{v=1}^{k} \boldsymbol{P}_{t}\left(\left\{N\left(A_{i}, \hat{x}\right)=n_{i}^{(v)} ; i=1, \ldots, m\right\} \mid\left\langle x_{v}\right\rangle\right)
\end{gathered}
$$

for $t \in T_{0}$, each decomposition $\left\{A_{1}, \ldots, A_{m}\right\}$ of $X$ with $A_{i} \in \mathfrak{A}, n_{i} \geqq 0, i=1, \ldots, m$, $m>0$, and each $\left\langle x_{1}, \ldots, x_{k}\right\rangle \in \hat{X}, k>0$, then it is called a branching transition function. Any Markov process $\left\{\hat{x}_{t} ; t \in T\right\}$ in $(\hat{X}, \hat{\mathfrak{A}})$ having such a transition function is called a Markov branching process.

We suppose to be given a branching transition function. We shall use the term "process" although we shall be dealing almost exclusively with this transition function and functionals constructed from it. Only the proof of Theorem 1' presupposes the existence of a corresponding process for discrete parameter or for a discrete skeleton imbedded in the parameter set. The existence of such a process is guaranteed without restrictions. The problem of constructing Markov branching processes will not be treated. (See e.g. [4].) 
1.2. Generating functionals. Denoting by $\mathscr{B}$ the Banach algebra of all bounded $\mathfrak{A}$-measurable, complex-valued functions $\xi$ on $X$ with norm $\|\xi\|:=\sup _{x \in X}|\xi(x)|$ and writing

$$
\begin{aligned}
f(\eta, \hat{x}): & :=1, & \hat{x} & =\theta, \\
& :=\prod_{v=1}^{n} \eta\left(x_{v}\right), & \hat{x} & =\left\langle x_{1}, \ldots, x_{n}\right\rangle,
\end{aligned}
$$

for $\eta \in \mathscr{B}$ and $\hat{x} \in \hat{X}$, we follow [8] in defining by

$$
F_{t}[\eta \mid \hat{x}]:=\int_{\hat{x}} f\left(\eta, \hat{x}^{\prime}\right) P_{t}\left(d \hat{x}^{\prime} \mid \hat{x}\right),
$$

at least on $\overline{\mathscr{S}}:=\{\eta \in \mathscr{B}:\|\eta\| \leqq 1\}$, the generating functional $F_{t}[\cdot \mid \hat{x}]$ associated with $\boldsymbol{P}_{t}(\cdot \mid \hat{x})$ for $t \in T_{0}$ and $\hat{x} \in \hat{X}$. By

$$
F_{t}[\eta]_{1}(x):=F_{t}[\eta \mid\langle x\rangle] \quad \forall \eta \in \overline{\mathscr{S}}, x \in X,
$$

we further define for each $t \in \boldsymbol{T}_{0}$ a generating mapping $F_{t}[\cdot]_{1}$ from $\overline{\mathscr{S}}$ into $\overline{\mathscr{S}}$ [9].

As illustrated by [9], generating functionals and mappings are a useful tool in the investigation of Markov branching processes. This is due mainly to the fact that the basic relations (1.1) and (1.2) for the branching transition function may be replaced by an equivalent set of simple functional relations for the associated generating functionals [8]:

The branching condition (1.2) is equivalent to

$$
\begin{aligned}
F_{t}[\eta \mid \hat{x}] & =1, & \hat{x} & =\theta, \\
& =\prod_{v=1}^{n} F_{t}\left[\eta \mid\left\langle x_{v}\right\rangle\right], & \hat{x} & =\left\langle x_{1}, \ldots, x_{n}\right\rangle,
\end{aligned}
$$

for $t \in T_{0}, \eta \in \overline{\mathscr{S}}$, and $\hat{x} \in \hat{X}$. (Proof. Taking any decomposition $\left\{A_{1}, \ldots, A_{m}\right\}$ of $X$ with $A_{i} \in \mathfrak{A}, i=1, \ldots, m, m>0$, inserting $\eta=\sum_{i=1}^{m} \lambda_{i} \chi_{A_{i}}$ into (1.5), and comparing coefficients of $\prod_{v=1}^{m} \lambda^{n_{v}}$ yields (1.2). This conclusion may be reversed utilizing the completeness of the set of stepfunctions in $\mathscr{B}$ and the continuity of generating functionals on $\overline{\mathscr{S}}$. The latter is obtained by means of a dominated convergence argument.)

Given (1.5), the Chapman-Kolmogorov equation (1.1) is equivalent to

$$
F_{t+s}[\eta \mid \hat{x}]=F_{s}\left[F_{t}[\eta]_{1} \mid \hat{x}\right]
$$

for $t, s \in T_{0}, \eta \in \overline{\mathscr{S}}$, and $\hat{x} \in \hat{X}$. (Propf. Representing $F_{t+s}[\eta \mid \hat{x}]$ as the limit of a sequence of Lebesgue-Stieltjes sums with finitely many terms each, replacing $\boldsymbol{P}_{t+s}(\cdot \mid \hat{x})$ according to (1.1), using dominated convergence to interchange integration and limit operation, finally applying (1.4) and (1.5) yields (1.6). By insertion of $\eta=\sum_{i=1}^{m} \lambda_{i} \chi_{A_{i}}$ into (1.6) and comparison of coefficients as above, (1.1) is recovered for a class of sets countably generating $\mathfrak{A}$ and thus, by a monotone convergence argument, for all $\hat{A} \in \hat{\mathfrak{A}}$.) 
According to [8] every generating functional is analytic [3] on

$$
\mathscr{S}:=\{\xi \in \mathscr{B}:\|\xi\|<1\} .
$$

The $k$ th Fréchet-differential of $F_{t}[\eta \mid \hat{x}]$ becomes

$$
\begin{array}{rlrl}
\delta^{k} F_{t}\left[\eta ; \xi_{1}, \ldots, \xi_{k} \mid \hat{x}\right] & =\int_{\hat{x}} f_{\xi_{1}, \ldots, \xi_{k}}\left(\eta, \hat{x}^{\prime}\right) \boldsymbol{P}_{t}\left(d \hat{x}^{\prime} \mid \hat{x}\right), & \\
f_{\xi_{1}, \ldots, \xi_{k}}\left(\eta, \hat{x}^{\prime}\right) & :=0, & N\left(X, \hat{x}^{\prime}\right)<k, \\
& :=\sum_{\left(i_{1}, \ldots, i_{n}\right)} \prod_{v=1}^{n} \xi_{v}\left(x_{i_{v}}\right), & \hat{x}^{\prime}=\left\langle x_{1}, \ldots, x_{n}\right\rangle, n=k, \\
& :=\frac{1}{(n-k) !} \sum_{\left(i_{1}, \ldots, i_{n}\right)} \prod_{v=1}^{k} \xi_{\nu}\left(x_{i_{v}}\right) \prod_{\mu=k+1}^{n} \eta\left(x_{i_{\mu}}\right), \\
\hat{x}^{\prime}=\left\langle x_{1}, \ldots, x_{n}\right\rangle, n>k,
\end{array}
$$

where summations extend over all permutations $\left(i_{1}, \ldots, i_{n}\right)$ of the numbers $1,2, \ldots, n[8]$.

In agreement with the assumptions in [9], even every generating mapping is analytic on $\mathscr{S}$. Given the analyticity of generating functionals, this follows immediately from the fact that any functional $F$ which is analytic and bounded by 1 on $\mathscr{S}$ satisfies

$$
\left|\frac{F[\eta+\lambda \xi]-F[\eta]}{\lambda}-\delta F[\eta ; \xi]\right| \leqq \frac{|\lambda| \cdot\|\xi\|^{2}}{(1-\|\eta\|)(1-\|\eta\|-|\lambda| \cdot\|\xi\|)}
$$

whenever $\|\eta\|+|\lambda| \cdot\|\xi\|<1$ with $\lambda$ a complex number and $\eta, \xi \in \mathscr{B}$. The estimate (1.8) is easily obtained by expanding $F[\eta+\lambda \xi]$ in powers of $\lambda$ and estimating the coefficients by means of Cauchy's integral formula [3]. (Remark. A more direct procedure yielding analyticity for generating functionals and mappings simultaneously starts from the explicit representation (1.4) with (1.3) and employs estimates as those used e.g. in the proof of [4, I, Lemma 0.1].)

Analyticity on $\mathscr{S}$ implies strong continuity on $\mathscr{S}$. An additional continuity property of generating functionals and mappings arises from the $\sigma$-additivity of the associated probability measures: Suppose $\xi_{t}, \xi \in \mathscr{B} \forall t \in \boldsymbol{T}$ and write $\pi-\lim _{t \rightarrow \infty} \xi_{t}$ $=\xi$ if $\lim _{t \rightarrow \infty} \xi_{t}(x)=\xi(x) \forall x \in X$. A functional $F$ defined at least on $\mathscr{D} \subset \mathscr{B}$ will be called $\pi$-sequentially continuous on $\mathscr{D}$ if $\lim _{v \rightarrow \infty} F\left[\xi_{v}\right]=F[\xi]$ for every sequence $\left\{\xi_{v}\right\}$ in $\mathscr{D}$ with $\pi-\lim _{v \rightarrow \infty} \xi_{v}=\xi \in \mathscr{D}$. (Remark. Since the space of complex numbers is a metric space, $\pi$-sequential continuity of $F$ on $\mathscr{D}$ entails $\lim _{t \rightarrow \infty} F\left[\xi_{t}\right]=\xi$ if $\xi_{t} \in \mathscr{D}$ $\forall t \in \boldsymbol{R}_{+}$and $\pi-\lim _{t \rightarrow \infty} \xi_{t}=\xi \in \mathscr{D}$.) It now follows from (1.4) by the dominated convergence theorem that $F_{t}[\cdot \mid \hat{x}]$ is $\pi$-sequentially continuous on $\overline{\mathscr{S}}$ for every $t \in \boldsymbol{T}_{0}$ and $\hat{x} \in \hat{X}$. An analogous argument applies to $\delta^{k} F_{t}\left[\eta ; \xi_{1}, \ldots, \xi_{k} \mid \hat{x}\right]$ on closed partial sets of $\mathscr{S} \otimes \mathscr{B}^{k}$. 
1.3. Factorial moment-functionals. For $\left(\xi_{1}, \ldots, \xi_{k}\right) \in \mathscr{B}^{k}, k>0$, and $\hat{x} \in \hat{X}$ let

$$
\begin{aligned}
N_{(k)}\left(\xi_{1}, \ldots, \xi_{k}, \hat{x}\right):=0, & N(X, \hat{x})<k, \\
:=\frac{1}{(n-k) !} \sum_{\left(i_{1} \ldots, i_{n}\right)} \prod_{v=1}^{k} \xi\left(x_{i_{v}}\right), & \hat{x}=\left\langle x_{1}, \ldots, x_{n}\right\rangle, n \geqq k,
\end{aligned}
$$

where the summation extends over all permutations $\left(i_{1}, \ldots, i_{n}\right)$ of $1,2, \ldots, n$. Employing this, we define by

$$
M_{(k)}^{t}\left[\xi_{1}, \ldots, \xi_{k} \mid \hat{x}\right]:=\int_{\hat{x}} N_{(k)}\left(\xi_{1}, \ldots, \xi_{k}, \hat{x}^{\prime}\right) \boldsymbol{P}_{t}\left(d \hat{x}^{\prime} \mid \hat{x}\right)
$$

the $k$ th factorial moment functional associated with $\boldsymbol{P}_{t}(\cdot \mid \hat{x})$ if the integral on the right exists for $\xi_{1}=\xi_{2}=\cdots=\xi_{k}=\mathbf{1}:=\chi_{X}$ and therewith for all $\left(\xi_{1}, \ldots, \xi_{k}\right) \in \mathscr{B}^{k}[8]$. By convention $M_{(1)}^{t}[\cdot \mid \hat{x}]=: M^{t}[\cdot \mid \hat{x}]$.

Every $k$ th factorial moment-functional is symmetric, $k$-linear, bounded, and therefore continuous on $\mathscr{B}^{k}$. In consequence of the $\sigma$-additivity of $\boldsymbol{P}_{t}(\cdot \mid \hat{x})$ it is also $\pi$-sequentially continuous on bounded regions in $\mathscr{B}^{k}$. Combining (1.7), (1.9), and (1.10), we have

$$
M_{(k)}^{t}\left[\xi_{1}, \ldots, \xi_{k} \mid \hat{x}\right]=\lim _{v \rightarrow \infty} \delta^{k} F_{t}\left[\eta_{v} ; \xi_{1}, \ldots, \xi_{k} \mid \hat{x}\right]
$$

for every sequence $\left\{\eta_{v}\right\}$ in $\overline{\mathscr{S}}$ with $\pi-\lim _{v \rightarrow \infty} \eta_{\nu}=\mathbf{1}$. Vice versa, if

$$
\lim _{v \rightarrow \infty} \delta^{k} F_{t}\left[\eta_{v} ; \mathbf{1}, \ldots, \mathbf{1} \mid \hat{x}\right]
$$

exists for some monotone sequence $\left\{\eta_{v}\right\}$ in $\mathscr{S}_{+}:=\{\xi \in \mathscr{S}: \xi(x) \geqq 0 \forall x \in X\}$ with $\pi$ - $\lim _{v \rightarrow \infty} \eta_{v}=1$, then $M_{(k)}^{t}\left[\xi_{1}, \ldots, \xi_{k} \mid \hat{x}\right]$ exists.

If $\sup _{x \in X} M_{(k)}^{t}[1, \ldots, 1 \mid\langle x\rangle]<\infty$, then

$$
M_{(k)}^{t}\left[\xi_{1}, \ldots, \xi_{k}\right]_{1}(x):=M_{(k)}^{t}\left[\xi_{1}, \ldots, \xi_{k} \mid\langle x\rangle\right] \quad \forall\left(\xi_{1}, \ldots, \xi_{k}\right) \in \mathscr{B}^{k}, x \in X,
$$

defines a mapping $M_{(k)}^{t}[\cdot]_{1}$ from $\mathscr{B}^{k}$ into $\mathscr{B}$ called the $k$ th factorial moment at $t$. Again $M^{t}[\cdot]_{1}:=M_{(1)}^{t}[\cdot]_{1}$, also $M^{t}:=M^{t}[\cdot]_{1}$. (Remark. Mullikin [9] used the strong limit s-lim $\operatorname{li1}_{\|\eta\| \rightarrow 0} \delta^{k} F_{t}\left[\eta ; \xi_{1}, \ldots, \xi_{k}\right]_{1}$ in place of the $k$ th factorial moment. It can be shown that the existence of $M_{(k+1)}^{t}[\cdot]_{1}$ is sufficient for the existence of this limit.)

We now turn to the expansion of generating functionals in factorial momentfunctionals. For any $\xi \in \mathscr{B}$ and $\hat{x}^{\prime} \in \hat{X}$

$$
\begin{aligned}
1-f\left(\xi, \hat{x}^{\prime}\right)= & -\left.\sum_{\nu=1}^{k-1} \frac{1}{\nu !} \frac{d^{v}}{d \lambda^{\nu}} f\left(1-\lambda(1-\xi), \hat{x}^{\prime}\right)\right|_{\lambda=0} \\
& -\frac{1}{(k-1) !} \int_{0}^{1}(1-\lambda)^{k-1} \frac{d^{k}}{d \lambda^{k}} f\left(1-\lambda(1-\xi), \hat{x}^{\prime}\right) d \lambda \\
= & \sum_{\nu=1}^{k} \frac{(-1)^{\nu+1}}{\nu !} N_{(v)}\left(1-\xi, \ldots, 1-\xi, \hat{x}^{\prime}\right)+\frac{(-1)^{k}}{k !} r_{(k)}(\xi)\left(1-\xi, \hat{x}^{\prime}\right)
\end{aligned}
$$


with

$$
\begin{aligned}
r_{(k)}(\xi)\left(\zeta, \hat{x}^{\prime}\right):=0, \quad N\left(X, \hat{x}^{\prime}\right) \leqq k, \\
:=\frac{1}{(n-k) !} \sum_{\left(i_{1}, \ldots, i_{n}\right)} \prod_{\nu=1}^{k} \zeta\left(x_{i_{v}}\right)\left(1-k \int_{0}^{1}(1-\lambda)^{k-1} \prod_{\mu=k_{k+1}}^{n}\left[1-\lambda\left(1-\xi\left(x_{i_{\mu}}\right)\right)\right] d \lambda\right), \\
\hat{x}^{\prime}=\left\langle x_{1}, \ldots, x_{n}\right\rangle, n>k,
\end{aligned}
$$

for $\zeta, \xi \in \mathscr{B}$. If $M_{(k)}^{t}[\cdot \mid \hat{x}]$ and thus $M_{(v)}^{t}[\cdot \mid \hat{x}], \nu \leqq k$, exists, we may integrate (1.12) for $\xi \in \overline{\mathscr{S}}$ with respect to $\boldsymbol{P}_{t}(\cdot \mid \hat{x})$ and obtain

(1.13) $1-F_{t}[\xi \mid \hat{x}]=\sum_{v=1}^{k} \frac{(-1)^{v+1}}{\nu !} M_{(v)}^{t}[1-\xi, \ldots, 1-\xi \mid \hat{x}]+\frac{(-1)^{k}}{k !} R_{(k)}^{t}(\xi)[1-\xi \mid \hat{x}]$

with

$$
R_{(k)}^{t}(\xi)[\zeta \mid \hat{x}]:=\int_{\hat{x}} r_{(k)}(\xi)\left(\zeta, \hat{x}^{\prime}\right) P_{t}\left(d \hat{x}^{\prime} \mid \hat{x}\right)
$$

for $\xi \in \overline{\mathscr{S}}$ and $\zeta \in \mathscr{B}$. Relation (1.13) generalizes expansions used in [5] for the case of finitely many types and refines an expression derived in [9] for $k=2$ on the basis of analyticity and more stringent moment assumptions.

For every $\xi \in \overline{\mathscr{S}}$ the functional $R_{(k)}^{t}(\xi)[\zeta \mid \hat{x}]$ is a bounded continuous monomial of degree $k$ in $\zeta \in \mathscr{B}$. It is $\pi$-sequentially continuous in $\xi \in \overline{\mathscr{S}}$ and in $\zeta$ on bounded regions in $\mathscr{B}$. Writing $\zeta \leqq \xi$ if $\zeta(x) \leqq \xi(x) \forall x \in X$ and defining 0 by $0(x):=0 \forall x \in X$, we have

$$
0 \leqq R_{(k)}^{t}\left(\xi^{\prime}\right)[\zeta \mid \hat{x}] \leqq R_{(k)}^{t}(\xi)\left[\zeta^{\prime} \mid \hat{x}\right] \leqq M_{(k)}^{t}\left[\zeta^{\prime}, \ldots, \zeta^{\prime} \mid \hat{x}\right]
$$

if $\mathbf{0} \leqq \xi \leqq \xi^{\prime} \leqq \mathbf{1}$ and $\mathbf{0} \leqq \zeta \leqq \zeta^{\prime}$. Combining this with

$$
(k+1) R_{(k)}^{t}(\xi)[1-\xi \mid \hat{x}]=M_{(k+1)}^{t}[1-\xi, \ldots, 1-\xi \mid \hat{x}]-R_{(k+1)}^{t}(\xi)[1-\xi \mid \hat{x}]
$$

we obtain

$$
\begin{aligned}
R_{(k)}^{t}(\xi)[1 \mid \hat{x}] & \leqq\left(1 /\|1-\xi\|^{k}\right) R_{(k)}^{t}(1-\|1-\xi\| 1)[\|1-\xi\| \mathbf{1} \mid \hat{x}] \\
& \leqq(1 /(k+1)) M_{(k+1)}^{t}[1, \ldots, 1 \mid \hat{x}]\|1-\xi\|
\end{aligned}
$$

whenever $\mathbf{0} \leqq \xi \leqq \mathbf{1}$ with $\xi \neq \mathbf{1}$.

If $M_{(k)}^{t}[\cdot]_{1}$ exists, then it follows from (1.14) that

$$
R_{(k)}^{t}(\xi)[\zeta]_{1}(x):=R_{(k)}^{t}(\xi)[\zeta \mid\langle x\rangle] \quad \forall \xi \in \overline{\mathscr{S}}, \zeta \in \mathscr{B}, x \in X,
$$

defines a mapping $R_{(k)}^{t}(\cdot)[\cdot]_{1}$ from $\overline{\mathscr{S}} \otimes \mathscr{B}$ into $\mathscr{B}$.

We conclude this section with functional relations derivable from (1.5) and (1.6) by means of (1.11). Equation (1.5) entails

$$
M^{t}\left[\xi \mid \hat{x}^{\prime}\right]=N_{(1)}\left(M^{t}[\xi]_{1}, \hat{x}^{\prime}\right) \quad \forall \xi \in \mathscr{B}, \hat{x}^{\prime} \in \hat{X},
$$

if $M^{t}[\cdot]_{1}$ exists. If in addition $M^{s}[\cdot \mid \hat{x}]$ exists, for some $\hat{x} \in \hat{X}$, then we may integrate (1.17) with respect to $\boldsymbol{P}_{s}(\cdot \mid \hat{x})$. Representing $M^{t}\left[\xi \mid \hat{x}^{\prime}\right]$ suitably as the limit of a 
sequenceof Lebesgue-Stieltjes sums, utilizing dominated convergence to interchange limit operation and integration with respect to $\boldsymbol{P}_{s}(\cdot \mid \hat{x})$, and finally applying $(1.1)$, we are lead to

$$
M^{t+s}[\xi \mid \hat{x}]=M^{s}\left[M^{t}[\xi]_{1} \mid \hat{x}\right] \quad \forall \xi \in \mathscr{B},
$$

which is due to Moyal [8].

Equation (1.18) may be obtained more directly by differentiating (1.6) in $\eta \in \mathscr{S}$,

$$
\delta F_{t+s}[\eta ; \xi \mid \hat{x}]=\delta F_{s}\left[F_{t}[\eta]_{1} ; \delta F_{t}[\eta ; \xi]_{1} \mid \hat{x}\right] \quad \forall \xi \in \mathscr{B},
$$

and subsequently letting $\eta$ approach $\mathbf{1}$. To justify this procedure even if

$$
\exists \eta \in \mathscr{S}:\left\|F_{t}[\eta]_{1}\right\|=1,
$$

we differentiate $F_{s, t}^{\varepsilon}[\eta \mid \hat{x}]:=F_{s}\left[\varepsilon F_{t}[\eta]_{1} \mid \hat{x}\right]$ for $\varepsilon \in(0,1)$ and afterwards let $\varepsilon$ tend to 1 , noting that $\delta F_{s}[\eta ; \xi \mid \hat{x}]$ has a continuous extension to $\overline{\mathscr{S}} \otimes \mathscr{B}$ if $M^{s}[\cdot \mid \hat{x}]$ exists, and using (1.8) to show that, for $(\eta, \xi) \in \mathscr{S} \otimes \mathscr{B}$ and $0<|\lambda|<(1-\|\eta\|) /\|\xi\|$,

$$
\begin{aligned}
& \left|\delta F_{s, t}^{\varepsilon}[\eta ; \xi \mid \hat{x}]-\delta F_{t+s}[\eta ; \xi \mid \hat{x}]\right| \\
& \quad \leqq|\lambda|^{-1}\left(\left|F_{s, t}^{\varepsilon}[\eta+\lambda \xi \mid \hat{x}]-F_{t+s}[\eta+\lambda \xi \mid \hat{x}]\right|+\left|F_{s, t}^{\varepsilon}[\eta \mid \hat{x}]-F_{t+s}[\eta \mid \hat{x}]\right|\right)+O_{\eta, \xi}(\lambda)
\end{aligned}
$$

with $\lim _{\lambda \rightarrow 0}\left|O_{\eta, \xi}(\lambda)\right|=0$ uniformly in $\varepsilon \in(0,1)$, that is,

$$
\lim _{\varepsilon \rightarrow 1} \delta F_{s, t}^{\varepsilon}[\eta ; \xi \mid \hat{x}]=\delta F_{t+s}[\eta ; \xi \mid \hat{x}] .
$$

Either method is applicable up to arbitrary order. The respective second order recurrence relation for factorial moments is

$$
M_{(2)}^{t+s}[\zeta, \xi \mid \hat{x}]=M^{s}\left[M_{(2)}^{t}[\zeta, \xi]_{1} \mid \hat{x}\right]+M_{(2)}^{s}\left[M^{t}[\zeta]_{1}, M^{t}[\xi]_{1} \mid \hat{x}\right]
$$

which holds for $\zeta, \xi \in \mathscr{B}$ in case the functionals and mappings involved on the right exist. If for some $\zeta, \xi \in \mathscr{B}_{+}:=\{\eta \in \mathscr{B}: \eta \geqq 0\}$ the formal procedure of deriving this relation yields infinity on the right, then $M^{t+s}[\cdot \mid \hat{x}]$ does not exist.

1.4. Quasi-positive regularity. If the first (factorial) moments associated with the given branching transition function exist, then according to (1.14) and (1.18) they form a semigroup of nonnegative, bounded, linear operators on $\mathscr{B}$. Certain spectral properties of this semigroup are strongly reflected in the asymptotic behaviour of the process and may therefore be used as a means of classification. We restrict ourselves to positively regular and quasi-positively regular processes. The former are defined as Markov branching processes whose moment semigroup exists and satisfies the following three conditions:

(M.1) For each $\xi \in \mathscr{B}_{+}$with $\xi \neq 0$ there exists a $\tau(\xi) \in T_{0}$ such that $\inf _{x \in X} M^{\tau(\xi)}[\xi \mid\langle x\rangle]>0$.

(M.2) For some $t_{0} \in T_{0}$ there is a compact operator $V$ on $\mathscr{B}$ such that $\left\|M^{t_{0}}-V\right\|<\rho^{t_{0}}:=\lim _{n \rightarrow \infty}\left\|M^{t_{0} n}[1]_{1}\right\|^{1 / n}$.

(M.3) If $T=R_{+}$, then $\left\|M^{t}[1]_{1}\right\|$ is bounded on bounded subsets of $T_{0}$, and $M^{t}[\xi \mid\langle x\rangle]$ is continuous in $t \in T_{0}$ for every $\xi \in \mathscr{B}$ and $x \in X$. 
Let $\mathscr{B}^{*}$ be the adjoint space of $\mathscr{B}$, further $\mathscr{B}_{+}^{*}:=\left\{\psi \in \mathscr{B}^{*}: \psi[\xi] \geqq 0 \forall \xi \geqq 0\right\}$, $\mathscr{B}_{+}^{\circ}:=\left\{\xi \in \mathscr{B}_{+}: \inf _{x \in X} \xi(x)>0\right\}$, and $\mathscr{B}_{+}^{\circ *}:=\left\{\psi \in \mathscr{B}_{+}^{*}: \psi[\xi]>0 \forall \xi \geqq \mathbf{0}, \xi \neq \mathbf{0}\right\}$. For real-valued $\eta, \xi \in \mathscr{B}$ we shall write $\eta \gg \xi$ if and only if $\eta-\xi \in \mathscr{B}_{+}^{\circ}$.

Proposition. A Markov branching process whose moment semigroup exists and satisfies (M.3) is positively regular if and only if $M^{t}$ may be represented in the form

$$
\begin{aligned}
M^{t} & =\rho^{t} P+Q_{t} & & \text { with } P Q_{t}=Q_{t} P=0, \\
P & =\varphi \varphi^{*}, & & \varphi \in \mathscr{B}_{+}^{\circ}, \varphi^{*} \in \mathscr{B}_{+}^{\circ *}, \varphi^{*}[\varphi]=1, \\
\left\|Q_{t}\right\| & \leqq K \alpha^{t}, & & 0 \leqq K<\infty, 0<\alpha<\rho,
\end{aligned}
$$

for all $t \in T_{0}$, where $\varphi^{*}$ is $\pi$-sequentially continuous on bounded regions in $\mathscr{B}$.

It is trivial that (M.1) and (M.2) are satisfied whenever (1.20) holds for all $t \in \boldsymbol{T}_{0}$. The converse conclusion has been established for $\boldsymbol{T}=\boldsymbol{Z}_{+}$in a more general setting by Karlin [6] in continuation of work done by Kreìn and Rutman [7], Yosida and Kakutani [10]:

The reflexivity of $\mathscr{B}$, the nonnegativity of $M^{1}$ with respect to the cone $\mathscr{B}_{+}$, and the nonemptiness of $\mathscr{B}_{+}^{\circ}$ and $\mathscr{B}_{+}^{\circ *}$ together entail the situation of $\rho$ in the point spectrum of $M^{1}$, as well as the existence of a nontrivial eigenvector $\varphi \in \mathscr{B}_{+}$and a nontrivial eigenfunctional $\varphi^{*} \in \mathscr{B}_{+}^{*}$ of $M^{1}$ associated with $\rho$. Assuming (M.1), we have $\rho>0, \varphi^{*} \in \mathscr{B}_{+}^{\circ *}$, and $\varphi \in \mathscr{B}_{+}^{\circ}$ which implies that $\rho$ is a simple eigenvalue [7].

If it is known in addition that $M^{1}$ possesses no spectral points of modulus $\rho$ except a finite number of discrete eigenvalues with finite multiplicity each, then $\rho$ is the only eigenvalue of modulus $\rho$ [6].

Given (M.2) and assuming the existence of a number $C<\infty$ such that $\left\|\rho^{-n} M^{n}\right\|$ $\leqq C \forall n>0$, the set of spectral points of $M^{1}$ with modulus $\rho$ does indeed consist of an at most finite number of discrete eigenvalues of finite multiplicity each, and the radius of the remaining part of the spectrum is smaller than $\rho$ [10].

These results are linked to establish (1.20) for $\boldsymbol{T}=Z_{+}$by the fact that $\rho>0$ and $\varphi \in \mathscr{B}_{+}^{\circ}$ combined imply the existence of a number $C<\infty$ such that $\left\|\rho^{-n} M^{n}\right\| \leqq C$ $\forall n>0$ [6].

The $\pi$-sequential continuity of $\varphi^{*}$ on bounded regions in $\mathscr{B}$ follows immediately from (1.20) and the $\pi$-sequential continuity of $M^{t}[\cdot \mid\langle x\rangle]$ on bounded regions in $\mathscr{B}$ for any $x \in X$.

We now reduce the continuous parameter case in the proof of the Proposition to the discrete parameter case.

Proof for continuous parameter. For each $\xi \in \mathscr{B}_{+}$with $\xi \neq 0$ and $s \in T_{0}$ with $s<\tau(\xi)$ there is by (M.1) a number $\varepsilon>0$ such that $M^{\tau(\xi)}[\xi]_{1} \geqq \varepsilon M^{\tau(\xi)-s}[\xi]_{1}$ and therewith

$$
M^{s}\left[M^{\tau(\xi)}[\xi]_{1}\right]_{1} \geqq \varepsilon M^{s}\left[M^{\tau(\xi)-s}[\xi]_{1}\right]_{1}=\varepsilon M^{\tau(\xi)}[\xi]_{1} \gg 0
$$

which entails $M^{t}[\xi]_{1} \gg \mathbf{0} \forall t \geqq \tau(\xi)$. 
Choosing for every $t \in \boldsymbol{T}\left(t_{0}\right):=\left\{r t_{0}: r>0\right.$ rational $\}$ a pair of positive integers $m(t), n(t)$ such that $t=m(t) t_{0} / n(t)$, we have $V_{t}:=M^{t_{0}{ }^{m(t)}}-\left(M^{t_{0}}-V\right)^{m(t)}$ as a compact operator satisfying

$$
\left\|M^{t n(t)}-V_{t}\right\|=\left\|\left(M^{t_{0}}-V\right)^{m(t)}\right\| \leqq\left\|M^{t_{0}}-V\right\|^{m(t)}<\rho^{t_{0} m(t)}=\rho^{t n(t)}
$$

for every $t \in \boldsymbol{T}\left(t_{0}\right)$.

Given a proof of the Proposition for discrete parameter, we may therefore write down a representation of the form

$$
\begin{aligned}
M^{t n} & =\rho^{t n} P_{t}+Q_{t, n} & & \text { with } P_{t} Q_{t, n}=Q_{t, n} P_{t}=0, \\
P_{t} & =\varphi_{t} \varphi_{t}^{*}, & & \varphi_{t} \in \mathscr{B}_{+}^{\circ}, \varphi_{t}^{*} \in \mathscr{B}_{+}^{\circ *}, \varphi_{t}^{*}\left[\varphi_{t}\right]=1, \\
\left\|Q_{t, n}\right\| & \leqq K_{t} \alpha_{t}^{n}, & & 0 \leqq K_{t}<\infty, 0<\alpha_{t}<\rho^{t},
\end{aligned}
$$

for $t \in \boldsymbol{T}\left(t_{0}\right)$ and $n \in Z_{+}-\{0\}$. Since this immediately yields

$$
\left\|P_{t_{0}}-P_{t}\right\| \leqq\left\|\rho^{-t n(t) v} Q_{t, n(t) v}\right\|+\left\|\rho^{-t_{0} m(t) v} Q_{t_{0}, m(t) v}\right\| \overrightarrow{v \rightarrow \infty} 0 \text { for } t \in T\left(t_{0}\right),
$$

we have even

$$
\begin{aligned}
M^{t} & =\rho^{t} P+Q_{t} & & \text { with } Q_{t} P=P Q_{t}=0, \\
P & =\varphi \varphi^{*}, & & \varphi \in \mathscr{B}_{+}^{\circ}, \varphi^{*} \in \mathscr{B}_{+}^{\circ *}, \varphi^{*}[\varphi]=1, \\
\left\|Q_{n t_{0}}\right\| & \leqq \text { const } \alpha^{n t_{0}}, & & 0<\alpha<\rho,
\end{aligned}
$$

for $t \dot{\epsilon} \boldsymbol{T}\left(t_{0}\right)$ and $n \in Z_{+}-\{0\}$, where $\varphi^{*}$ is again $\pi$-sequentially continuous on bounded regions in $\mathscr{B}$.

Consistently with (1.21) we now define $Q_{t}:=M^{t}-\rho^{t} P \forall t \in T_{0}$. Using $P M^{t}$ $=M^{t} P=\rho^{t} P \forall t \in T\left(t_{0}\right)$ which follows from (1.21), and taking into account (M.3), the $\pi$-sequential continuity of $\varphi^{*}$, and the fact that $\boldsymbol{T}\left(t_{0}\right)$ is dense in $T_{0}$, we have $P M^{t}=M^{t} P=\rho^{t} P \forall t \in T_{0}$. This implies $Q_{t} P=P Q_{t}=0 \forall t \in T_{0}$. It also entails $Q_{t} Q_{s}$ $=Q_{t+s} \forall t, s \in T_{0}$. Since it follows from (M.3) that $\left\|Q_{t}\right\|$ is bounded on bounded subsets of $\boldsymbol{T}_{0}$, we thus arrive at $\left\|Q_{t}\right\|=\left\|Q_{n t_{0}} Q_{s}\right\| \leqq\left\|Q_{n t_{0}}\right\| \cdot\left\|Q_{s}\right\| \leqq$ const $\alpha^{n t_{0}}$ for $t=n t_{0}+s \in\left(n t_{0},(n+1) t_{0}\right), n \in Z_{+}-\{0\}$, and consequently $\left\|Q_{t}\right\| \leqq K \alpha^{t} \forall t \in T_{0}$ with $K<\infty$.

Subsequently the conditions (M.1), (M.2), and (M.3) will not be used explicitly but only through (1.20). In fact, we shall not always need the full strength of (1.20); the disturbing restriction $\varphi^{*} \in \mathscr{B}_{+}^{\circ *}$ is not essential for the main results. We therefore introduce so-called quasi-positively regular processes defined as Markov branching processes whose moment semigroup exists and satisfies

$$
\text { representation }(1.20) \text { with } \varphi^{*} \in \mathscr{B}_{+}^{*} \text { instead of } \varphi^{*} \in \mathscr{B}_{+}^{\circ *} \text {. }
$$

A positively or quasi-positively regular process is called critical if $\rho=1$.

II. Limit theorems. In the following we are concerned with the asymptotic behaviour of critical, positively or quasi-positively regular Markov branching 
processes. As indicated above the treatment applies to processes with continuous parameter as well as to processes with discrete parameter. Apart from differences in the moment assumptions the theorems and their corollaries extend results given for discrete parameter by Mullikin [9]. Lemmata 2 and 4 generalize results proven for finite set of types and discrete parameter by Joffe and Spitzer [5].

Recalling $F_{t}[0 \mid\langle x\rangle]=\boldsymbol{P}_{t}(\{\theta\} \mid\langle x\rangle) \forall t \in \boldsymbol{T}_{0}, x \in X$, we define the asymptotic extinction function

$$
\boldsymbol{q}:=\pi-\lim _{t \rightarrow \infty} F_{t}[\mathbf{0}]_{1}
$$

[8]. It always exists, since by (1.4) and (1.6) we have $\mathbf{0} \leqq F_{t^{\prime}}[0]_{1} \leqq F_{t^{\prime \prime}}[0]_{1} \leqq \mathbf{1}$ for $t^{\prime} \leqq t^{\prime \prime}$.

Given quasi-positive regularity, $F_{t}[\xi]_{1}$ certainly cannot be independent of $\xi$ on $\overline{\mathscr{S}}$ for any $t \in T_{0}$. However, $F_{t}[\xi]_{1}-F_{t}[0]_{1}$ may be linear and not vanishing identically in $\xi$ for all $t \in \boldsymbol{T}_{0}$, that is, we may have $\boldsymbol{P}_{t}(N(X, \hat{x}) \leqq 1 \mid\langle x\rangle)=1 \forall t \in \boldsymbol{T}_{0}, x \in X$ with $\exists t \in \boldsymbol{T}_{0}: \boldsymbol{P}_{t}(N(X, \hat{x})=0 \mid\langle x\rangle)=1 \forall x \in X$. In this case

$$
F_{t}[\xi]_{1}=F_{t}[\mathbf{0}]_{1}+M^{t}[\xi]_{1} \quad \forall t \in T_{0}, \xi \in \mathscr{B} .
$$

For a critical, quasi-positively regular process this entails

$$
\lim _{t \rightarrow \infty} \boldsymbol{P}_{t}(N(A, \hat{x})=1 \mid\langle x\rangle)=\varphi(x) \varphi^{*}\left[\chi_{A}\right] \quad \forall A \in \mathfrak{A}, x \in X,
$$

and

$$
1=q+\varphi \varphi^{*}[1] .
$$

Taking into account $\varphi^{*}[\varphi]=1$, the latter yields $\varphi^{*}[q]=0$. Hence there exists an $A \in \mathfrak{A}$ with $\varphi^{*}\left[\chi_{A}\right]=0$ such that $q(x)=0 \forall x \in X-A$. Since $F_{t}[0]_{1}$ is monotone increasing in $t$, this implies $\boldsymbol{P}_{t}(\{\theta\} \mid\langle x\rangle)=0 \forall t \in T_{0}, x \in X-A$.

We are left with those processes for which $F_{t}[\xi]_{1}-F_{i}[0]_{1}$ is nonlinear in $\xi$ for some $t \in \boldsymbol{T}_{0}$. A Markov branching process having this property is called nonsingular.

THEOREM 1. For every nonsingular, critical, positively regular process

$$
\mathrm{s}-\lim _{t \rightarrow \infty} F_{t}[\xi]_{1}=1 \text { uniformly in } \xi \in \overline{\mathscr{S}} \text {. }
$$

Proof. Employing (1.6), $\pi$-sequential coñtinuity of $F_{t}[\cdot]_{1},(1.13)$ for $k=1$, and (1.14), we obtain

$$
1-q=1-F_{t}[q]_{1} \leqq M^{t}[1-q]_{1} \quad \forall t \in T_{0} .
$$

By (1.20) with $\rho=1$ we have

$$
\varphi^{*}\left[M^{t}[1-q]_{1}-(1-q)\right]=0 \quad \forall t \in T_{0} .
$$

In view of $\varphi^{*} \in \mathscr{B}_{+}^{\circ *}$ therefore

$$
1-q=1-F_{t}[q]_{1}=M^{t}[1-q]_{1} \quad \forall t \in T_{0} .
$$


Again by (1.20) with $\rho=1$ this implies

$$
\exists c \in \boldsymbol{R}_{+}: \mathbf{1}-\boldsymbol{q}=c \varphi
$$

and on account of (1.13) also

$$
R_{(1)}^{t}(q)[1-q]_{1}=0 \quad \forall t \in T_{0} .
$$

For $\|\boldsymbol{q}\|<1$ the latter can hold only if $\left.\boldsymbol{P}_{t}\left(X^{(n)} \mid\langle x\rangle\right)=0 \forall t \in \boldsymbol{T}_{0}, n\right\rangle 1, x \in X$, which is incompatible with the assumed nonsingularity of the process. Hence $\|\boldsymbol{q}\|=1$. Because of $\varphi \gg 0$ this entails $c=0$, that is, $q=1$. Consequently

$$
\lim _{t \rightarrow \infty} \varphi^{*}\left[1-F_{t}[0]_{1}\right]=0,
$$

where it has been used that $\varphi^{*}$ is $\pi$-sequentially continuous on bounded regions in $\mathscr{B}$. Combining (1.6), (1.13) for $k=1,(1.14)$, and (1.20) with $\rho=1$, we have

$$
\begin{aligned}
\left\|\mathbf{1}-F_{t+s}[0]_{1}\right\| & =\left\|\mathbf{1}-F_{s}\left[F_{t}[0]_{1}\right]_{1}\right\| \\
& \leqq\left\|M^{s}\left[\mathbf{1}-F_{t}[0]_{1}\right]\right\| \leqq\|\varphi\| \varphi^{*}\left[\mathbf{1}-F_{t}[\mathbf{0}]_{1}\right]+K \alpha^{s}
\end{aligned}
$$

with $\alpha<1$. By (2.2) therefore s-lim $\lim _{t \rightarrow \infty} F_{t}[0]_{1}=1$. The estimate

$$
\begin{aligned}
\left\|\mathbf{1}-F_{t}[\xi]_{1}\right\| & \left.\leqq\left\|\mathbf{1}-F_{t}[\mathbf{0}]_{1}\right\|+\| F_{t}|| \xi \mid\right]_{1}-F_{t}[\mathbf{0}]_{1} \| \\
& \leqq 2\left\|\mathbf{1}-F_{t}[\mathbf{0}]_{1}\right\| \quad \forall \xi \in \overline{\mathscr{S}}
\end{aligned}
$$

completes the proof.

To secure a similar result for quasi-positively regular processes, we introduce another condition on the first moments:

(M.4) There exist, for some $t^{\prime} \in T_{0}$, a bounded measure $G(\cdot) \mid \mathfrak{A}$ and a bounded function $m^{t^{\prime}}(\cdot \mid \cdot) \mid X \otimes X$ such that

$$
M^{t^{\prime}}\left[\chi_{A} \mid\langle x\rangle\right]=\int_{A} m^{t^{\prime}}(y \mid x) G(d y) \quad \forall A \in \mathfrak{A}, x \in X .
$$

(Note. It follows from the additivity and $\pi$-sequential continuity of $M^{t}[\cdot \mid \hat{x}]$ that $M^{t}\left[\chi_{A} \mid \hat{x}\right]$ is a measure on $\mathfrak{A}$ for each $t \in T_{0}$ and $\hat{x} \in \hat{X}$.)

For real-valued $\xi, \zeta \in \mathscr{B}$ we shall write $\xi>\zeta$ if and only if $\xi(x)>\zeta(x) \forall x \in X$.

THEOREM 1'. If a critical, quasi-positively regular process satisfies (M.4) and $\exists u \in T_{0}: F_{u}[0]_{1}>\mathbf{0}$, then

$$
\text { s- } \lim _{t \rightarrow \infty} F_{t}[\xi]_{1}=1 \text { uniformly in } \xi \in \overline{\mathscr{S}} \text {. }
$$

The proof up to $q=\mathbf{1}$ consists in the slightly adapted proofs of [3, III, Theorems $11.1,11.2$, and 11.3]. Having established $q=1$, we may employ the same argument that was used in the proof of Theorem 1 .

Recalling (1.5), we immediately have

COROLlaRY 1.1. On the hypotheses of Theorem 1 as well as those of Theorem 1'

$$
\lim _{t \rightarrow \infty} \boldsymbol{P}_{t}(\{\theta\} \mid \hat{x})=1
$$

uniformly in $\hat{x} \in X^{(n)}$ for every $n>1$. 
We continue with several additional conditions and auxiliary statements preparing further limit theorems.

LEMMA 1. For every quasi-positively regular process

$$
\left|F_{t}[\xi]_{1}\right|<\mathbf{1} \quad \forall t \in \boldsymbol{T}_{0}, \xi \in \mathscr{S} .
$$

Proof. From the relations defining generating mappings we read off $\left|F_{t}[\xi]_{1}\right|$ $\leqq F_{t}[|\xi|]_{1}$ and $F_{t}[|\xi|]_{1}-F_{t}[\mathbf{0}]_{1} \leqq\|\xi\|\left(1-F_{t}[0]_{1}\right)$, that is, $1-\left|F_{t}[\xi]_{1}\right| \geqq\left(1-F_{t}[|\xi|]_{1}\right)$ $\geqq(1-\|\xi\|)\left(1-F_{t}[0]_{1}\right)$ for $t \in T_{0}, \xi \in \mathscr{S}$. By $F_{t}[0 \mid\langle x\rangle]=\boldsymbol{P}_{t}(\{\theta\} \mid\langle x\rangle)$ we should have $M^{t}[1 \mid\langle x\rangle]=0$ if $1-F_{t}[0 \mid\langle x\rangle]=0$. On the other hand, (1.20') implies $M^{t}[1]_{1}$ $\geqq\|\varphi\|^{-1} M^{t}[\varphi]_{1} \gg \mathbf{0} \forall t \in \boldsymbol{T}_{0}$. Hence $1-\left|F_{t}[\xi]_{1}\right|>\mathbf{0} \forall t \in \boldsymbol{T}_{0}, \xi \in \mathscr{S}$.

For the following we replace (M.4) by a more stringent condition:

(M.4') Condition (M.4) with $G(A)=\varphi^{*}\left[\chi_{A}\right] \forall A \in \mathfrak{A}$.

We also impose

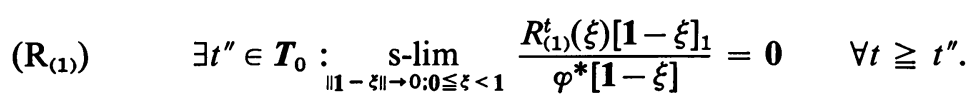

For positively regular processes with finite $X$, condition $\left(\mathrm{R}_{(1)}\right)$ is automatically satisfied. In case of a quasi-positively regular process with general set of types there are conditions on the first and second factorial moments dominating $\left(R_{(1)}\right)$ : For example, let (M.4') be satisfied and assume that for some $s \in \boldsymbol{T}_{0}$ there exists a bounded function $m_{(2)}^{\text {s }}(1 ; \cdot \mid \cdot) \mid X \otimes X$ such that

$$
M_{(2)}^{s}\left[1, \chi_{A} \mid\langle x\rangle\right]=\int_{A} m_{(2)}^{s}(1 ; y \mid x) \varphi^{*}\left[\chi_{d y}\right] \quad \forall A \in \mathfrak{A}, x \in X,
$$

then $\left(\mathbf{R}_{(1)}\right)$ is satisfied. This can be seen as follows:

By (M.4') and (1.18) there exist a $t^{\prime} \in T_{0}$ and a real-valued function $C_{1}(\cdot) \mid T_{0}$ such that

$$
M^{t}\left[\chi_{A}\right]_{1} \leqq C_{1}(t) \varphi^{*}\left[\chi_{A}\right] \mathbf{1} \quad \forall t \geqq t^{\prime}, A \in \mathfrak{A} .
$$

Given $\left(1.20^{\prime}\right)$, the existence of $M_{(2)}^{s}[\cdot, \cdot]_{1}$ entails the existence of $M_{(2)}^{t}[\cdot, \cdot]_{1}$ for all $t \in \boldsymbol{T}_{0}$. This is easily verified, using (1.19) and the nonnegativity of factorial moments. Setting $M^{0}[\xi]_{1}:=\xi \forall \xi \in \mathscr{B}$ and choosing some integer $n>0$ such that $n s \geqq t^{\prime}$, we obtain from (1.19)

$$
\begin{aligned}
M_{(2)}^{n s+t}\left[1, \chi_{A}\right]_{1} & =M_{(2)}^{t}\left[M^{n s}[1]_{1}, M^{n s}\left[\chi_{A}\right]_{1}\right]_{1} \\
+ & \sum_{v=1}^{n} M^{(v-1) s+t}\left[M_{(2)}^{s}\left[M^{(n-v) s}[1]_{1}, M^{(n-v) s}\left[\chi_{A}\right]_{1}\right]_{1}\right]_{1} \quad \forall t \in T_{0}, A \in \mathfrak{U} .
\end{aligned}
$$

From this with $\left(1.20^{\prime}\right)$ and $(2.4)$

$$
\begin{aligned}
M_{(2)}^{n s+t}\left[1, \chi_{A}\right]_{1} \leqq M_{(2)}^{t}[1,1]_{1}(\|P\|+K) \rho^{n s} C_{1}(n s) \varphi^{*}\left[\chi_{A}\right] \\
+\rho^{t+(2 n-1) s} \sum_{v=1}^{n} \rho^{-v s}(\|P\|+K)^{2} \sup _{x, y \in X}\left|m_{(2)}^{s}(1 ; y \mid x)\right| \varphi^{*}\left[\chi_{A}\right] 1 \quad \forall t \in T_{0}, A \in \mathfrak{X} .
\end{aligned}
$$


Hence there exists a real-valued function $C_{2}(\cdot) \mid T_{0}$ such that

$$
M_{(2)}^{n s+t}[1,1-\xi]_{1} \leqq C_{2}(t) \varphi^{*}[1-\xi] 1 \quad \forall t \in T_{0}, \xi \in \overline{\mathscr{S}}_{+}:=\overline{\mathscr{S}} \cap \mathscr{B}_{+} .
$$

As a consequence of (1.15), (1.14), and (2.5), condition $\left(R_{(1)}\right)$ is satisfied.

LEMMA 2. If a quasi-positively regular process satisfies (M.4'), $\left(\mathrm{R}_{(1)}\right)$, and $\mathrm{s}-\lim _{t \rightarrow \infty} F_{t}[0]_{1}=1$, then

$$
\text { s-lim } \frac{1-F_{t}[\xi]_{1}}{\varphi^{*}\left[1-F_{t}[\xi]_{1}\right]}=\varphi \quad \text { uniformly in } \xi \in \mathscr{S}_{+} .
$$

Proof. 1. By (M.4') and $\left(1.20^{\prime}\right)$

$$
\begin{aligned}
\left\|M^{t^{\prime}+t}\left[\chi_{A}\right]_{1}-\rho^{t^{\prime}+t} P\left[\chi_{A}\right]\right\| & =\left\|Q_{t}\left[Q_{t^{\prime}}\left[\chi_{A}\right]_{1}\right]_{1}\right\| \\
& \leqq K \alpha^{t}\left(\sup _{x, y \in X}\left|m^{t^{\prime}}(y \mid x)\right|+\rho^{t^{\prime}}\|\varphi\|\right) \varphi^{*}\left[\chi_{A}\right] \quad \forall t \in T_{0}, A \in \mathfrak{A} .
\end{aligned}
$$

Taking into account $0<\alpha<\rho$ and $\varphi \gg 0$, it follows that there exists a nonnegative function $\varepsilon(\cdot) \mid T_{0}$ with

$$
\lim _{t \rightarrow \infty} \varepsilon(t)=0
$$

such that

$$
(1-\varepsilon(t)) \rho^{t} P[\eta] \leqq M^{t}[\eta]_{1} \leqq(1+\varepsilon(t)) \rho^{t} P[\eta] \quad \forall t>t^{\prime}, \eta \in \mathscr{B}_{+} .
$$

Given $\left(R_{(1)}\right)$, there is for each $t \in T_{0}$ a nonnegative functional $g_{t}[\cdot] \mid \mathscr{S}_{+}$with

$$
\lim _{\|1-\zeta\| \rightarrow 0} g_{t}[\zeta]=0 \quad \forall t \in T_{0}
$$

such that

$$
\mathbf{0} \leqq R_{(1)}^{t}(\zeta)[1-\zeta]_{1} \leqq g_{t}[\zeta] \rho^{t} P[1-\zeta] \quad \forall t \geqq t^{\prime \prime}, \zeta: 0 \leqq \zeta<1 .
$$

2. Combining (1.6) and (1.13), we have

$$
\begin{aligned}
\mathbf{1}-F_{t}[\xi]_{1} & =1-F_{s}\left[F_{t-s}[\xi]_{1}\right]_{1} \\
& =M^{s}\left[\mathbf{1}-F_{t-s}[\xi]_{1}\right]_{1}-R_{(1)}^{s}\left(F_{t-s}[\xi]_{1}\right)\left[1-F_{t-s}[\xi]_{1}\right]_{1} \quad \forall s, t \in T_{0}, \xi \in \overline{\mathscr{S}} .
\end{aligned}
$$

From this, by (2.8), (2.3), and (2.10),

$$
\begin{aligned}
&\left(1-\varepsilon(s)-g_{s}\left[F_{t-s}[\xi]_{1}\right]\right) \rho^{s} P\left[1-F_{t-s}[\xi]_{1}\right] \\
& \leqq 1-F_{t}[\xi]_{1} \leqq(1+\varepsilon(s)) \rho^{s} P\left[1-F_{t-s}[\xi]_{1}\right] \\
& \forall s>\max \left(t^{\prime}, t^{\prime \prime}\right), t>s, \xi \in \mathscr{S}_{+}
\end{aligned}
$$

and, by applying $\varphi^{*}$, also

$$
\begin{aligned}
(1-\varepsilon(s)- & \left.g_{s}\left[F_{t-s}[\xi]_{1}\right]\right) \rho^{s} \varphi^{*}\left[1-F_{t-s}[\xi]_{1}\right] \\
& \leqq \varphi^{*}\left[1-F_{t}[\xi]_{1}\right] \leqq(1+\varepsilon(s)) \rho^{s} \varphi^{*}\left[1-F_{t-s}[\xi]_{1}\right] \\
& \forall s>\max \left(t^{\prime}, t^{\prime \prime}\right), t>s, \xi \in \mathscr{S}_{+} .
\end{aligned}
$$


In view of (2.3) and $\varphi^{*} \in \mathscr{B}_{+}^{*}$ with $\varphi^{*}[\varphi]=1$ we have $\varphi^{*}\left[1-F_{t-s}[\xi]_{1}\right]>0 \forall t-s \in T_{0}$, $\xi \in \mathscr{S}_{+}$. By (2.11) and (2.12) therefore

$$
\begin{aligned}
-\frac{2 \varepsilon(s)+g_{s}\left[F_{t-s}[\xi]_{1}\right]}{1+\varepsilon(s)} \varphi \leqq \frac{1-F_{t}[\xi]_{1}}{\varphi^{*}\left[1-F_{t}[\xi]_{1}\right]}-\varphi & \leqq \frac{2 \varepsilon(s)+g_{s}\left[F_{t-s}[\xi]_{1}\right]}{1-\varepsilon(s)-g_{s}\left[F_{t-s}[\xi]_{1}\right]} \varphi \\
& \forall s>\max \left(t^{\prime}, t^{\prime \prime}\right), t>s, \xi \in \mathscr{S}_{+} .
\end{aligned}
$$

Recalling (2.7), (2.9), and the fact that s- $\lim _{t \rightarrow \infty} F_{t}[0]_{1}=1$ entails s- $\lim _{t \rightarrow \infty} F_{t}[\xi]_{1}=1$ uniformly in $\xi \in \overline{\mathscr{S}}$, this yields (2.6).

We further restrict ourselves to processes satisfying

$\left(\mathrm{M}_{(2)}\right)$ The second factorial moment exists at each $t \in \boldsymbol{T}_{0}$ and, in case $\boldsymbol{T}=\boldsymbol{R}_{+}$, we have $\lim _{t \rightarrow 0} \varphi^{*}\left[M_{(2)}^{t}[1,1]_{1}\right]=0$.

Given (1.20') and the existence of $M_{(2)}^{t}[1,1]_{1}$ for some $t \in T_{0}$ it follows by use of (1.19) and the nonnegativity of factorial moments that $\left\|M_{(2)}^{t}[1,1]_{1}\right\|$ is bounded on bounded partial sets of $T_{0}$. Because of the $\pi$-sequential continuity of $\varphi^{*}$ on bounded regions it is therefore sufficient for $\lim _{t \rightarrow 0} \varphi^{*}\left[M_{(2)}^{t}[1,1]_{1}\right]=0$ that there exists an $A \in \mathfrak{A}$ with $\varphi^{*}\left[\chi_{X-A}\right]=0$ such that $\lim _{t \rightarrow 0} M_{(2)}^{t}[1,1 \mid\langle x\rangle]=0 \forall x \in A$.

LEMMA 3. If a critical, quasi-positively regular process satisfies $\left(\mathrm{M}_{(2)}\right)$, then

$$
\mu:=(1 / 2 t) \varphi^{*}\left[M_{(2)}^{t}[\varphi, \varphi]_{1}\right]
$$

is constant as a function of $t \in T_{0}$.

Proof. By (1.19) and $\left(1.20^{\prime}\right)$ with $\rho=1$

$$
\varphi^{*}\left[M_{(2)}^{(k+1) s}[\varphi, \varphi]_{1}\right]=\varphi^{*}\left[M_{(2)}^{k s}[\varphi, \varphi]_{1}\right]+\varphi^{*}\left[M_{(2)}^{s}[\varphi, \varphi]_{1}\right]
$$

for $k \in Z_{+}-\{0\}$ and $s \in T_{0}$. From this

$$
\varphi^{*}\left[M_{(2)}^{n / m}[\varphi, \varphi]_{1}\right]=n \varphi^{*}\left[M_{(2)}^{1 / m}[\varphi, \varphi]_{1}\right]=(n / m) \varphi^{*}\left[M_{(2)}^{1}[\varphi, \varphi]_{1}\right]
$$

with $n, m \in \boldsymbol{Z}_{+}-\{0\}$ for $\boldsymbol{T}=\boldsymbol{R}_{+}$, and $n \in \boldsymbol{Z}_{+}-\{0\}, m=1$ for $\boldsymbol{T}=\boldsymbol{Z}_{+}$. In case $\boldsymbol{T}=\boldsymbol{R}_{+}$, it follows from $\lim _{t \rightarrow 0} \varphi^{*}\left[M_{(2)}^{t}[1,1]_{1}\right]=0$ that $\lim _{t \rightarrow 0} \varphi^{*}\left[M_{(2)}^{t}[\varphi, \varphi]_{1}\right]=0$, and from this with (1.19) and $\left(1.20^{\prime}\right)$ that $\varphi^{*}\left[M_{(2)}^{t}[\varphi, \varphi]_{1}\right]$ is continuous in $t \in \boldsymbol{T}_{0}$. Combination of these facts yields the proposed constancy of $\mu$.

As a last condition we postulate

$\left(\mathrm{R}_{(2)}\right)$ For every monotone nondecreasing sequence $\left\{\xi_{v}\right\}$ in $\overline{\mathscr{S}}_{+}$with $\mathrm{s}-\lim _{v \rightarrow \infty} \xi_{v}=1$ there are $\sigma, \varepsilon \in T_{0}$ such that

$$
\lim _{v \rightarrow \infty} \varphi^{*}\left[R_{(2)}^{t}\left(\xi_{v}\right)[1]_{1}\right]=0 \quad \text { uniformly in } t \in[\sigma, \sigma+\varepsilon] \cap T_{0} .
$$

For a quasi-positively regular process with discrete parameter this condition is automatically satisfied if the second factorial moments exist. In case $\boldsymbol{T}=\boldsymbol{R}_{+}$, we certainly have $\varphi^{*}\left[R_{(2)}^{t}\left(\xi_{v}\right)[1]_{1}\right] \rightarrow 0$ for every $t \in T_{0}$ but not necessarily uniform convergence on some nondegenerate $t$-interval. By (1.16), however, such a uniform convergence is guaranteed at least if $\sup _{t \in[\sigma, \sigma+\varepsilon]} \sup _{x \in A} M_{(3)}^{t}[1,1,1 \mid\langle x\rangle]<\infty$ for 
some $\sigma, \varepsilon>0$ and an $A \in \mathfrak{U}$ with $\varphi^{*}\left[\chi_{X-A}\right]=0$. Given $\left(1.20^{\prime}\right)$, it is sufficient for the latter that $M_{(3)}^{t}[1,1,1]_{1}$ exists for some $t \in T_{0}$.

LEMMA 4. If a critical, quasi-positively regular process satisfies the conditions of Lemma 2 and in addition to these also $\left(\mathrm{M}_{(2)}\right)$ and $\left(\mathrm{R}_{(2)}\right)$, then

$$
\lim _{t \rightarrow \infty} \frac{1}{t}\left[\frac{1}{\varphi^{*}\left[1-F_{t}[\xi]_{1}\right]}-\frac{1}{\varphi^{*}[1-\xi]}\right]=\mu \quad \text { for every in } \xi \in \mathscr{S}_{+}
$$

and uniformly on $\left\{\xi \in \mathscr{S}_{+}:\|1-\xi\| / \varphi^{*}[1-\xi]<c,\|1-\xi\|<\varepsilon / c\right\}$ for every $c<\infty$ and some $\varepsilon>0$.

Proof. 1. By $\varphi^{*} \in \mathscr{B}_{+}^{*}, \varphi^{*}[\varphi]=1,(2.3)$, and (1.6) we have $\varphi^{*}[1-\zeta]>0$ and $\varphi^{*}\left[1-F_{t}[\zeta]_{1}\right]>0 \forall t \in T_{0}$ whenever $\zeta \in \mathscr{S}_{+}$or $\zeta=F_{s}[\xi]_{1}$ with $\xi \in \mathscr{S}_{+}$and $s \in T_{0}$. For any such $\zeta$ we may therefore write down the identity

$$
\begin{aligned}
& 1-\frac{1}{\varphi^{*}[1-\zeta]}\left(\varphi^{*}[1-\zeta]-\varphi^{*}\left[1-F_{t}[\zeta]_{1}\right]\right)\left[\frac{1}{\varphi^{*}\left[1-F_{t}[\zeta]_{1}\right]}-\frac{1}{\varphi^{*}[1-\zeta]}\right] \\
&=\frac{1}{\left(\varphi^{*}[1-\zeta]\right)^{2}}\left(\varphi^{*}[1-\zeta]-\varphi^{*}\left[1-F_{t}[\zeta]_{1}\right]\right) \quad \forall t \in T_{0}
\end{aligned}
$$

which, by means of $(1.13)$ for $k=2,\left(1.20^{\prime}\right)$ with $\rho=1$, and $\Delta[\zeta]:=(1-\zeta) / \varphi^{*}[1-\zeta]$, is transformed into

$$
\left(2-\varphi^{*}[1-\zeta]\left(\varphi^{*}\left[M_{(2)}^{t}[\Delta[\zeta], \Delta[\zeta]]_{1}\right]-\varphi^{*}\left[R_{(2)}^{t}(\zeta)[\Delta[\zeta]]_{1}\right]\right)\right)
$$

$$
\begin{gathered}
\cdot\left[\frac{1}{\varphi^{*}\left[1-F_{t}[\zeta]_{1}\right]}-\frac{1}{\varphi^{*}[1-\zeta]}\right] \\
=\varphi^{*}\left[M_{(2)}^{t}[\Delta[\zeta], \Delta[\zeta]]_{1}\right]-\varphi^{*}\left[R_{(2)}^{t}(\zeta)[\Delta[\zeta]]_{1}\right] \quad \forall t \in T_{0} .
\end{gathered}
$$

2. Employing $h_{s}[\xi]:=\Delta\left[F_{s}[\xi]_{1}\right]-\varphi \forall s \in \boldsymbol{T}_{0}, \xi \in \mathscr{S}_{+}$together with $\mu$ from (2.13), and $\varphi \gg \mathbf{0}$, we have

$$
\begin{aligned}
\mid \varphi^{*}\left[M _ { ( 2 ) } ^ { t } \left[\Delta\left[F_{s}[\xi]_{1}\right]\right.\right. & \left.\left., \Delta\left[F_{s}[\xi]_{1}\right]\right]_{1}\right]-2 t \mu \mid \\
& =\left|\varphi^{*}\left[2 M_{(2)}^{t}\left[\varphi, h_{s}[\xi]\right]_{1}+M_{(2)}^{t}\left[h_{s}[\xi], h_{s}[\xi]\right]_{1}\right]\right| \\
& \leqq \varphi^{*}\left[M_{(2)}^{t}[\varphi, \varphi]_{1}\right]\left(c_{1}\left\|h_{s}[\xi]\right\|+c_{2}\left\|h_{s}[\xi]\right\|^{2}\right) \quad \forall t, s \in T_{0}, \xi \in \mathscr{S}_{+}
\end{aligned}
$$

with some constants $c_{1}<\infty, c_{2}<\infty$. Since according to Lemma 2

$$
\lim _{s \rightarrow \infty}\left\|h_{s}[\xi]\right\|=0 \quad \text { uniformly in } \xi \in \mathscr{S}_{+} \text {, }
$$

this yields

$$
\lim _{s \rightarrow \infty} \varphi^{*}\left[M_{(2)}^{t}\left[\Delta\left[F_{s}[\xi]_{1}\right], \Delta\left[F_{s}[\xi]_{1}\right]_{1}\right]=2 t \mu\right.
$$

uniformly in $\xi \in \mathscr{S}_{+}$as well as in $t$ on every bounded partial set of $\boldsymbol{T}_{0}$.

Using (1.14), (2.1) or $\left(2.1^{\prime}\right)$ respectively, $\left(\mathrm{R}_{(2)}\right)$, and (2.17), we get

$$
\begin{aligned}
\lim _{s \rightarrow \infty}\left|\varphi^{*}\left[R_{(2)}^{t}\left(F_{s}[\xi]_{1}\right)\left[\Delta\left[F_{s}[\xi]_{1}\right]\right]_{1}\right]\right| & \\
& \leqq \lim _{s \rightarrow \infty} \varphi^{*}\left[R_{(2)}^{t}\left(F_{s}[0]_{1}\right)[1]_{1}\right]\left(\|\varphi\|+\left\|h_{s}[\xi]\right\|\right)^{2}=0
\end{aligned}
$$

uniformly in $\xi \in \mathscr{S}_{+}$and in $t \in[\sigma, \sigma+\varepsilon] \cap T_{0}$ with suitable $\sigma, \varepsilon>0$. 
3. It follows from (2.16) by substituting $\zeta=F_{s}[\xi]_{1}$ and applying (1.6), (2.18), and (2.19) that

$$
\lim _{s \rightarrow \infty}\left[\frac{1}{\varphi^{*}\left[1-F_{t+s}[\xi]_{1}\right]}-\frac{1}{\varphi^{*}\left[1-F_{s}[\xi]_{1}\right]}\right]=t \mu
$$

uniformly in $\xi \in \mathscr{S}_{+}$and in $t \in[\sigma, \sigma+\varepsilon] \cap \boldsymbol{T}_{0}$. Defining the integral-valued function $n(t) \mid\left\{t \in T_{0}: t \geqq \sigma\right\}$ by $n(t) \sigma \leqq t<(n(t)+1) \sigma \forall t \geqq \sigma$, setting $\delta(t):=t / n(t) \forall t \geqq \sigma$ and $F_{0}[\xi]_{1}:=\xi \forall \xi \in \mathscr{S}$, and utilizing the uniformity of convergence in (2.20), we finally obtain

$$
\begin{aligned}
\lim _{t \rightarrow \infty} \frac{1}{t}\left[\frac{1}{\varphi^{*}\left[1-F_{t}[\xi]_{1}\right]}-\frac{1}{\varphi^{*}[1-\xi]}\right] \\
\quad=\lim _{t \rightarrow \infty} \frac{1}{n(t) \delta(t)} \sum_{k=0}^{n(t)-1}\left[\frac{1}{\varphi^{*}\left[1-F_{(k+1) \delta(t)}[\xi]_{1}\right]}-\frac{1}{\varphi^{*}\left[1-F_{k \delta(t)}[\xi]_{1}\right]}\right]=\mu
\end{aligned}
$$

for every $\xi \in \mathscr{S}_{+}$and with the proposed uniformity.

The following results are of interest only for $\mu>0$ although they formally remain true if $\mu \rightarrow 0$, as long as s-lim $\lim _{t \rightarrow \infty} F_{t}[0]_{1}=1$ is assured. By (2.13) and (1.10) we have $\mu>0$ if and only if for some $s \in T_{0}$ there exists an $A \in \mathfrak{A}$ with $\varphi^{*}\left[\chi_{A}\right]>0$ such that $\boldsymbol{P}_{s}\left(X^{(n)} \mid\langle x\rangle\right)>0 \forall x \in A$ for at least one $n>1$ or, equivalently, that $F_{s}[\xi \mid\langle x\rangle]$ $-F_{s}[0 \mid\langle x\rangle]$ is nonlinear in $\xi$ for $x \in A$. A quasi-positively regular process having this property will be called $\varphi^{*}$-nonsingular.

THEOREM 2. Let a critical, quasi-positively regular process be $\varphi^{*}$-nonsingular and satisfy $\left(\mathrm{M} .4^{\prime}\right),\left(\mathbf{R}_{(1)}\right),\left(\mathbf{M}_{(2)}\right)$, and $\left(\mathbf{R}_{(2)}\right)$. In case this process is not positively regular, suppose further that $\exists u \in T_{0}: F_{u}[0]_{1}>\mathbf{0}$. Then

$$
\mathrm{s}-\lim _{t \rightarrow \infty} t\left(1-F_{t}[\xi]_{1}\right)=\varphi / \mu \quad \forall \xi \in \mathscr{S}_{+} .
$$

Proof. Taking into account Theorem 1 and Theorem 1' respectively, this result is obtained immediately by combining Lemma 2 and Lemma 4.

COROllary 2.1. On the assumptions of Theorem 2

$$
\lim _{t \rightarrow \infty} t \boldsymbol{P}_{t}(\hat{X}-\{\theta\} \mid \hat{x})=N_{(1)}(\varphi, \hat{x}) / \mu
$$

uniformly in $\hat{x} \in X^{(n)}$ for every $n>0$.

Proof. Using (1.5) and proceeding as in (1.12), we get

$$
\begin{aligned}
t \boldsymbol{P}_{t}(\hat{X}-\{\theta\} \mid \hat{x}) & =t\left(1-F_{t}[0 \mid \hat{x}]\right) \\
& =N_{(1)}\left(t\left(1-F_{t}[0]_{1}\right), \hat{x}\right)-r_{(1)}\left(F_{t}[0]_{1}\right)\left(t\left(1-F_{t}[0]_{1}\right), \hat{x}\right)
\end{aligned}
$$

which by (2.21) leads to (2.22).

The remaining two results which involve conditional expectations and distributions refer to the respective regular versions defined through the given transition function. 
COROLlARY 2.2. On the assumptions of Theorem 2

$$
\lim _{t \rightarrow \infty} E\left(\frac{N_{(1)}\left(\xi, \hat{x}_{t}\right)}{t} \mid \hat{x}_{t} \neq \theta, \hat{x}_{0}=\hat{x}\right)=\mu \varphi^{*}[\xi]
$$

uniformly in $\hat{x} \in X^{(n)}$ for every $n>0$ and $\xi \in \mathscr{B}$.

Proof. Relation (2.23) is obtained from

$$
M^{t}[\xi \mid \hat{x}]=\boldsymbol{P}_{t}(\hat{X}-\{\theta\} \mid \hat{x}) \boldsymbol{E}\left(N_{(1)}\left(\xi, \hat{x}_{t}\right) \mid \hat{x}_{t} \neq \theta, \hat{x}_{0}=\hat{x}\right)
$$

by means of (2.22), (1.17), and $\left(1.20^{\prime}\right)$ with $\rho=1$.

REMARK. The monotony of $F_{t}[0]_{1}$ with respect to $t$ allows $\left(\mathrm{R}_{(2)}\right)$ to be eliminated from the assumptions of Corollaries 2.1 and 2.2.

THEOREM 3. Let a critical, quasi-positively regular process be $\varphi^{*}$-nonsingular and satisfy $\left(\mathrm{M} .4^{\prime}\right),\left(\mathrm{R}_{(1)}\right),\left(\mathrm{M}_{(2)}\right)$, and $\left(\mathrm{R}_{(2)}\right)$. In case this process is not positively regular, suppose further that $\exists u \in T_{0}: F_{u}[0]_{1}>0$. Then, for any decomposition $\left\{A_{1}, \ldots, A_{n}\right\}$ of $X$ with $A_{i} \in \mathfrak{A}, i=1, \ldots, n, n>0$, and any $\hat{x} \in \hat{X}-\{\theta\}$, the conditional distribution of the vector

$$
\frac{1}{t}\left(N\left(A_{1}, \hat{x}_{t}\right), \ldots, N\left(A_{n}, \hat{x}_{t}\right)\right) \text { given } \hat{x}_{t} \neq \theta, \hat{x}_{0}=\hat{x}
$$

converges, as $t \rightarrow \infty$, to the distribution of a vector of the form

$$
\left(\varphi^{*}\left[\chi_{A_{1}}\right], \ldots, \varphi^{*}\left[\chi_{A_{n}}\right]\right) \cdot W
$$

with

$$
\boldsymbol{P}(W \leqq z)=\chi_{(0, \infty)}(z)(1-\exp [-z / \mu]) \quad \forall z \in(-\infty, \infty) .
$$

Proof. For any $\xi:=\sum_{i=1}^{n} \lambda_{i} \chi_{A_{i}}$ with $\lambda_{i}>0, i=1, \ldots, n$, and $\xi_{t}:=\exp [-\xi / t]$ $\forall t \in T_{0}$ we have

$$
\lim _{t \rightarrow \infty} t \varphi^{*}\left[1-\xi_{t}\right]=\varphi^{*}[\xi]
$$

According to Lemma 2

$$
1-F_{t}[\eta]_{1}=\varphi^{*}\left[1-F_{t}[\eta]_{1}\right]\left(\varphi+h_{t}[\eta]\right) \quad \forall t \in T_{0}, \eta \in \mathscr{S}_{+}
$$

with

$$
\lim _{t \rightarrow \infty}\left\|h_{t}[\eta]\right\|=0 \quad \text { uniformly in } \eta \in \mathscr{S}_{+} \text {, }
$$

and by Lemma 4

$$
\varphi^{*}\left[1-F_{t}[\eta]_{1}\right]=\frac{\varphi^{*}[1-\eta]}{1+t \varphi^{*}[1-\eta]\left(\mu+k_{t}[\eta]\right)} \quad \forall t \in T_{0}, \eta \in \mathscr{S}_{+}
$$

with

$$
\lim _{t \rightarrow \infty} k_{t}[\eta]=0 \quad \text { uniformly in } \eta \in\left\{\xi_{s}: s>s_{0}\right\}
$$


for some $s_{0} \in \boldsymbol{T}_{0}$. Combining these five relations we obtain

$$
\mathrm{s}-\lim _{t \rightarrow \infty} t\left(1-F_{t}\left[\xi_{t}\right]_{1}\right)=\frac{\varphi^{*}[\xi] \varphi}{1+\varphi^{*}[\xi] \mu} \text {. }
$$

Using (1.5), (1.12), (2.22), (2.27), and (2.1) or (2.1') respectively, the limit of the Laplace-Stieltjes transform of the conditional distribution of (2.24), as $t \rightarrow \infty$, becomes

$$
\begin{aligned}
\lim _{t \rightarrow \infty} E(\exp & {\left.\left[-\sum_{i=1}^{n} \lambda_{i} \frac{N\left(A_{i}, \hat{x}_{t}\right)}{t}\right] \mid \hat{x}_{t} \neq \theta, \hat{x}_{0}=\hat{x}\right) } \\
= & \lim _{t \rightarrow \infty} \frac{F_{t}\left[\xi_{t} \mid \hat{x}\right]-F_{t}[\mathbf{0} \mid \hat{x}]}{1-F_{t}[\mathbf{0} \mid \hat{x}]}=1-\frac{\lim _{t \rightarrow \infty} t\left(1-F_{t}\left[\xi_{t} \mid \hat{x}\right]\right)}{\lim _{t \rightarrow \infty} t\left(1-F_{t}[\mathbf{0} \mid \hat{x}]\right)} \\
= & 1-\frac{\mu}{N_{(1)}(\varphi, x)} \lim _{t \rightarrow \infty}\left(N_{(1)}\left(t\left(1-F_{t}\left[\xi_{t}\right]_{1}\right), \hat{x}\right)-r_{(1)}\left(F_{t}\left[\xi_{t}\right]_{1}\right)\left(t\left(1-F_{t}\left[\xi_{t}\right]_{1}\right), \hat{x}\right)\right) \\
& =1-\frac{\mu}{N_{(1)}(\varphi, \hat{x})} \frac{N_{(1)}(\varphi, \hat{x}) \varphi^{*}[\xi]}{1+\varphi^{*}[\xi] \mu}=\frac{1}{1+\mu \varphi^{*}[\xi]}
\end{aligned}
$$

which is the Laplace-Stieltjes transform of the distribution of (2.25) with (2.26). On account of the continuity theorem this entails the proposed convergence in distribution.

\section{REFERENCES}

1. V. P. Cistjakov, Two limit theorems for branching processes with $n$ types of particles, Teor. Verojatnost. i Primenen. 4 (1959), 477-478 = Theor. Probability Appl. 4 (1959), 436-437.

2. T. E. Harris, The theory of branching processes, Springer-Verlag, Berliñ; Prentice-Hall, Englewood Cliffs, N. J., 1963. MR 29 \#664.

3. E. Hille and R. S. Phillips, Functional analysis and semi-groups, rev. ed., Amer. Math. Soc. Colloq. Publ., vol. 31, Amer. Math. Soc., Providence, R. I., 1957. MR 19, 664.

4. N. Ikeda, M. Nagasawa and S. Watanabe, Branching Markov processes. I, II, III, J. Math. Kyoto Univ. 8 (1968), 233-278, 365-410; ibid. 9 (1969), 95-160. MR 38 \#764; MR 38 \#6677; MR 39 \#7680.

5. A. Joffe and F. Spitzer, On multitype branching processes with $p \leqq 1$, J. Math. Anal. Appl. 19 (1967), 409-430. MR 35 \#3760.

6. S. Karlin, Positive operators, J. Math. Mech. 8 (1959), 907-937. MR 22 \#4965.

7. M. G. Krein and M. A. Rutman, Linear operators leaving invariant a cone in a Banach space, Uspehi Mat. Nauk 3 (1948), 3-95; English transl., Amer. Math. Soc. Transl. (1) 26 (1950). MR 10, 256; MR 12, 341.

8. J. E. Moyal, The general theory of stochastic population processes, Acta Math. 108 (1962), 1-31. MR $26 \# 5616$.

9. T. W. Mullikin, Limiting distributions for critical multitype branching processes with discrete time, Trans. Amer. Math. Soc. 106 (1963), 469-494. MR 26 \#1931.

10. K. Yosida and S. Kakutani, Operator-theoretical treatment of Markoff's process and mean ergodic theorem, Ann. of Math. (2) 42 (1941), 188-228. MR 2, 230.

InStitut für Mathematische Statistik, Úniversität KaRLSRUhe, KaRLSRUHe, GermanY 